\title{
Acompanhamento do envelhecimento humano em centro de convivência
}

| ${ }^{1}$ Maria Martha Duque de Moura, ${ }^{2}$ Renato Peixoto Veras |

Resumo: Este artigo discute o papel dos centros de convivência no acompanhamento do envelhecimento humano. A partir de revisão bibliográfica, exploramos história, experiências e possibilidades dos centros de convivência. Percebemos "senicultura" como cultivo, mas também controle social do idoso. Evitando o controle, a rede de cuidados pode nortear suas ações pela autoavaliação do idoso e sua percepção de qualidade de vida. Enfatizamos a importância dos vínculos, a relação cuidador-cuidado como espaço de transformação da maneira de lidar com o envelhecimento. Propomos centros de cuidado como estratégia para evitar o isolamento dos idosos e oportunizar a convivência intergeracional.

> Palavras-chave: idoso; promoção de saúde; centro de convivência.

\author{
1 Instituto da Mulher, da Criança \\ e do Adolescente Fernandes \\ Figueira (IFF/Fiocruz). Rio de \\ Janeiro-RJ, Brasil (martha@iff. \\ fiocruz.br). \\ 2 Universidade Aberta da \\ Terceira Idade, Universidade do \\ Estdo do Rio de Janeiro. Rio de \\ Janeiro-RJ, Brasil (unativeras@ \\ gmail.com).
}

Recebido em: 20/12/2015 Aprovado em: 22/04/2016 
O envelhecimento populacional é fenômeno mundial. Contamos com 600 milhões de idosos no mundo - três vezes mais do que há 50 anos, três vezes menos do que teremos em 2050 (MANDELBAUM, 2013). O crescimento do número de idosos, aliado à diminuição do número de filhos, resulta na atual transição demográfica. No Brasil, o ritmo desta transição é acelerado (CAMARANO, 2006). Ademais, a população considerada idosa está também envelhecendo, aumentando a proporção daqueles com mais de 80 anos (WICHMANN et al., 2013).

Diante deste quadro, a Organização Mundial da Saúde divulgou em 2002 sua proposta de envelhecimento ativo, atenta à manutenção da saúde, com foco na autonomia e independência funcional do idoso (OMS, 2005). O crescente custo dos cuidados em saúde, determinado pela introdução de novas tecnologias, mas também pelo aumento da prevalência de doenças crônicas e de sua duração, suscita hoje discussão e exige reorganização do cuidado do idoso.

Nos países da Europa Ocidental e América do Norte, as pirâmides etárias são geralmente altas e estreitas, devido à baixa taxa de natalidade e à esperança de vida elevada. Ao longo do tempo, esses países desenvolveram estratégias para cuidar dos idosos. No Brasil, esta preocupação é crescente e a rede de cuidados de idosos está em desenvolvimento.

A dificuldade de acesso aos serviços de saúde faz com que muitos idosos só recebam os primeiros cuidados em estágios avançados de adoecimento. Assim, missão importante dos centros de convivência é melhorar a saúde e a qualidade de vida dos idosos, proporcionando-lhes atividades que contribuam para que sejam menos afetados por doenças crônicas, prevenindo incapacidade e recuperando autonomia com programas de reabilitação (VERAS; CAMARGO JR., 1995; VERAS, 2012a).

O objetivo deste artigo é explorar, no campo da Saúde Coletiva, possibilidades de promoção da saúde em centros de convivência para idosos. São destacados aspectos de promoção e psicossociais do envelhecimento, enfatizando a informação e vínculos. A partir do ciclo vital humano, é traçado paralelo entre o cuidado da criança e do idoso, isto é, entre a puericultura e a senicultura. Apontam-se questões de funcionamento e integração dos centros de convivência com a rede de proteção e cuidados. 
O estudo é baseado em revisão bibliográfica e sua discussão subsidiada pela experiência profissional dos autores. A revisão da literatura proporcionou síntese de conhecimento para possíveis incorporações dos resultados na prática cotidiana de centros de convivência (SOUZA et al., 2010; MENDES et al., 2008). As perguntas norteadoras do estudo foram: qual o papel dos centros de convivência no acompanhamento do envelhecimento humano descrito na produção científica? Quais suas possíveis ações?

A revisão bibliográfica realizada na base de dados PUBMED (7/2014 e 7/2015), utilizando os descritores "idoso", "centro de convivências" e "promoção de saúde" ("elderly", "senior centers" and "health promotion"), identificou 126 artigos (n.126). Após leitura de todos os títulos e resumos, foram excluídos artigos que não tinham como tema principal a experiência em centros de convivências para idosos (n.30/126) ou que analisavam grupos populacionais específicos (n.6/126).

Foram selecionados para leitura na íntegra os artigos que analisavam experiências de centros de convivência para idosos com promoção de saúde de forma ampla, metodologias de avaliação e sua integração da rede de cuidados (n.26/126). Destes, foram escolhidos oito artigos com análises mais abrangentes e dois que referiam relações intergeracionais em centro de convivência, citados no presente artigo, que apresenta 65 referências bibliográficas (10/65). Entre os artigos que abordavam prevenção e monitoramento de doenças específicas (n.14/126), ou atividades específicas (n.50/126), seis foram também selecionados, ilustrando possíveis ações nesses centros (6/65). A revisão Pubmed foi complementada com busca de literatura citada nos artigos selecionados (3/65).

A discussão é informada pela experiência profissional da primeira autora como pediatra nas creches da Fundação Oswaldo Cruz (Fiocruz/MS) e na maternidade do Instituto Fernandes Figueira/Fiocruz, com observação e atenção aos vínculos, o que gerou a inclusão de algumas referências (5/65). Sua formação mais recente (2013-2014) no curso de pós-graduação em Gerontologia e Geriatria da Universidade Aberta da Terceira Idade da Universidade do Estado do Rio de Janeiro (http://www.unatiuerj.com.br/cursosSuperior.htm), coordenado pelo segundo autor, com estágio prático em centro de convivência, resultou na inclusão de sugestôes de bibliografia feita por especialistas (SOUZA et al., 
2010), professores desse curso. São, sobretudo, referências nacionais (36/65), mas também algumas de língua francesa (5/65).

\section{Discussão}

Neste artigo, empregamos o termo idoso, do francês personne âgée, que nos parece ser mais respeitoso, comparado a "velho" (associado à aposentadoria, dependência e incapacidade) ou "terceira idade" (troisième age), que designa principalmente aposentados dinâmicos, representando novo mercado no turismo, produtos de beleza e saúde (PEIXOTO, 2000a). Idoso refere-se ao grupo de pessoas com mais de 60 anos. São jovens velhos (60 a 70 anos), velhos ( 80 a 90 anos) e muito velhos (acima de 90 anos) com autonomia e independência bem variadas.

Propomos o termo senicultura, aludindo àquele usado para o "cultivo" da infância (puericultura), para nos referirmos às ações de promoção de saúde e prevenção de doenças, recorrentes no cuidado de idosos.

Neste artigo exploraremos o centro de convivência de idosos, equipamento coletivo que promove sua socialização através de atividades de lazer, educacionais e de promoção de saúde.

\section{Os centros de convivência}

As experiências internacionais de acompanhamento do envelhecimento são singulares, culturalmente determinadas, impossíveis de reproduzir. Entretanto, ao observá-las, percebemos que podem se apresentar como possibilidades, fontes de inspiração (CACHIONI, 1999; MOLINA, 2011; WICK, 2012; VERAS, 2012b; PEIXOTO, C 2000).

Países como Inglaterra e França construíram políticas e estratégias para lidar com o envelhecimento da população. Cachioni (1999), ao discutir a origem das universidades da terceira idade, apresenta alguns exemplos. Na França, o modelo precursor existia desde os anos 1960, oferecendo atividades culturais e de incentivo à sociabilidade. Nessa época, não havia preocupação com programas dirigidos à educação permanente, para a saúde ou assistência jurídica; havia apenas a promoção de atividades ocupacionais e lúdicas.

$\mathrm{Na}$ Itália, agentes gerontológicos são formados para atuar em programas de atualização cultural, visando a prevenção de doenças, instrumentalização do idoso e discussão do envelhecimento. $\mathrm{Na}$ China, centros de recreação oferecem 
programas de saúde, jantares comunitários, jogos e várias modalidades esportivas.

Já alguns programas na Escandinávia procuram fortalecer a autoestima do idoso e sua inserção ou reinserção no mercado de trabalho. Na Irlanda existe um projeto intergeracional, o Agelink, que produz atividades entre escolas para crianças e idosos (CACHIONI, 1999).

$\mathrm{Na}$ Espanha, Molina (2011) fez revisão bibliográfica sobre centros para mayores e centro-dia, apontando que a frequência a centros-dia para mayores diminui o consumo de medicamentos e consultas médicas. Ao oferecer rotina, atividades de caráter repetitivo, buscam sensibilizar o idoso para o autocuidado; e colônia de férias para idosos, viagens em grupo e visitas domiciliares ampliam suas possibilidades. A avaliação do grau de dependência e a eliminação de barreiras para a vida independente são observadas. As rodas de conversas, discussão de rotinas garantem o protagonismo dos idosos e profissionais, ampliando a comunicação da rede e criando entorno social seguro. Acesso e continuidade da frequência são a meta estabelecida através de um contrato de frequência e duração (MOLINA, 2011).

No Canadá, Hawranik \& Pangman (2002) analisaram experiência dos centros de convivência (senior's wellness centers) a partir de grupos focais com os idosos que frequentam o centro e os profissionais. O serviço de enfermagem, por exemplo, pode lhes ser útil, mesmo que não estejam doentes, abrindo portas para o autocuidado através da informação, resultando em diminuição de visitas aos médicos e diminuição de custos. As prioridades desses centros são: uso apropriado dos serviços de saúde; prevenção de quedas; uso seguro de medicações; cuidados em saúde mental. Nessa experiência, após seis meses foi registrada melhora significativa no estilo de vida, e nas informaçôes sobre temas como medicamentos, quedas, dietas, abuso e o suporte jurídico ao idoso (HAWRANIK; PANGMAN, 2002).

Nos EUA, o programa Elderhostel promove, desde 1975, atividades como aulas, conferências, viagens de turismo. Naquele país também é comum os idosos participarem de atividades voluntárias. Wick (2012) discute o esvaziamento, a baixa adesão da população a alguns desses centros: não basta a construção desses centros, suas atividades devem coincidir com a demanda da população local.

Metodologias para avaliar o impacto da experiência dos centros de convivência na vida dos participantes e sua sustentabilidade como equipamento coletivo têm 
sido desenvolvidas. Utilizam-se instrumentos como medida da qualidade de vida, autorrelato em grupos focais, número de dias de cama, frequência e duração dos períodos de vigília e sono, escalas de depressão, número de idas aos hospitais ou consultas médicas, que são indicadores de saúde, e funcionalidade física e mental (WALLACE, 1998; PARDASANI, 2010; COBERLEY et al., 2011; RILL, 2011).

$\mathrm{Na}$ França, as municipalidades (mairies) oferecem no seu Centre Culturel atividades regulares para os idosos residentes: jogos, oficinas, informática, etc. Na prefeitura de Puteaux, na Grande Paris, um dia dedicado aos idosos acontece com mutirão para rastreio visual, auditivo, dentário, cardiovascular e análise de sangue (diabete e colesterol). Essa municipalidade agencia serviços de assistência de vida (banho e higiene) e/ou ajuda instrumental (faxina da casa, alimentação e compras) com despesa rateada entre prefeitura e idoso. $\mathrm{Na}$ prefeitura estão registradas as associações para manutenção em domicílio daquela municipalidade (PUTEAUX, 2015).

\section{O caso do Brasil}

Segundo histórico de Cachioni (1999), no Brasil, com o gradativo aumento da população idosa, surgiram alguns programas educacionais voltados principalmente ao lazer. A primeira experiência brasileira de educação para adultos maduros e idosos foi implementada pelo Serviço Social do Comércio (SESC). Os Grupos de Convivência surgiram na década de 1960, com programação elaborada com base em programas de lazer. Eram assistencialistas, pois não ofereciam instrumentos necessários para os sujeitos recuperarem a autonomia desejada. A partir da década de 1980, as universidades começaram a abrir espaço educacional para a população idosa e para profissionais interessados no estudo das questôes do envelhecimento, predominando a oferta de programas de ensino, saúde e lazer (CACHIONI, 1999).

O centro de convivências de idosos refere-se a diferentes espaços que promovem atividades regulares para idosos, do futebol semanal na beira de rio até o centro com 65 oficinas dentro de uma universidade. Grupos de convivência ligados ao Programa de Saúde da Família, lar de idosos e centros de convivência estão vinculados à prefeitura do Rio de Janeiro. Nem sempre os profissionais que ali coordenam atividades têm proposta integrada à rede de cuidados. 
O censo SUAS (Sistema Único da Assistência Social) iniciou em 2014 coleta de dados de estrutura e funcionamento dos 7.890 centros de convivência públicos e privados registrados no Brasil. No primeiro censo, foram coletadas informaçôes de 442 centros de convivência ( $5 \%$ dos cadastrados), cerca da metade (44,8\%) destinada a atividades com idosos; os outros se destinam a outras faixas etárias. Não há referência específica sobre atividades intergeracionais.

Ao discutir assistência aos idosos com foco no monitoramento, cuidado e prevenção, ao invés do protagonismo do hospital, Veras propõe uma linha de cuidado com cinco níveis hierárquicos. O centro de convivência, nível 2 de hierarquia na linha de cuidado de idosos, é espaço de promoção da saúde e de educação, e disponibiliza informações sobre hábitos saudáveis, ações preventivas e avaliação geriátrica ampla - AGA (VERAS, 2011). Os três primeiros níveis (acolhimento, núcleo integrado de cuidado, ou seja, centros de convivência, centro-dia, ambulatório de baixa complexidade e o ambulatório especializado) são considerados unidades leves, de baixo custo e grande resolutividade. Os dois últimos, cuidado de curta duração (emergência, hospital-dia, atenção domiciliar, hospice) e cuidado de longa duração (instituição de longa permanência, residência assistida, unidade de reabilitação) são considerados de custo elevado, ou pesado.

No Sistema Único de Saúde do Brasil (SUS), muitas vezes iniciativas de cuidados de idosos estão ligadas ao Programa de Saúde da Família (BRASIL, 2006). Integralidade, vínculo e acolhimento na gestão do cuidado necessitam mais ampla discussão (GOMES; PINHEIRO, 2005).

Centros de convivências para idosos têm sido criados pelas operadoras de planos de saúde a partir da publicação, pela Agência Nacional de Saúde Suplementar (ANS), do Plano de cuidado para idosos na saúde suplementar. $\mathrm{O}$ documento propóe incentivos para estimular a mudança da lógica de assistência, criando espaços para promoção em saúde para idosos, tais como os centros de convivência (ANS, 2010). Publicou-se também resolução que incentiva a participação de beneficiários de planos de saúde em programas de envelhecimento ativo, com a possibilidade de descontos nas mensalidades (VERAS, 2011). O custo elevado do plano de saúde suplementar e a postura médico-sanitarista das visitas domiciliares que propõe-polícia sanitária restringem suas possibilidades. 


\section{Possibilidades}

Diante da necessidade de construção de múltiplas estratégias de suporte àqueles que necessitam de proteção e cuidados, perguntamo-nos: a quem cabe o cuidado da criança e do idoso? Responder a esta questão supõe traçar paralelos entre ambos.

$\mathrm{Na}$ linha do cuidado no ciclo de vida humana, a infância e a velhice guardam proximidades. Crianças pequenas, pessoas com doenças crônicas, e pessoas idosas, todos que têm limitada autonomia e independência, necessitam de cuidados. Equipamentos coletivos, como creches e centros de convivências, podem ampliar as possibilidades de suporte e acompanhamento, as abordagens intergeracionais de cuidado, e alargar padrōes de integração social. A ampliação das redes de apoio e dos recursos para lidar com limitações enriquece a experiência do viver (MOURA, 2001).

Há muito a família é encarregada do sustento de crianças e idosos. Com o advento das aposentadorias no século XX, ela passou a compartilhar essas responsabilidades com as empresas e o Estado. A construção de redes de cuidado representa um alívio considerável às famílias, aumentando suas chances de fazer face a suas responsabilidades. Contudo, o poder está presente em todas as relaçôes humanas (FOUCAULT, 1985). Como legitimar o outro evitando uma relação de tutela; como evitar que o cuidado se efetive como exercício de dominação? É preciso administrar bem o espaço de poder presente em qualquer relação no sentido de recusar estratégias de dominação (BARROS, 2008). A relação dialógica, o protagonismo dos sujeitos, a negociação e corresponsabilidade fazem toda a diferença (GOMES; PINHEIRO, 2005).

Discutiremos adiante a questão da qualidade da relação cuidador-idoso e as possibilidades de transformação nas relações de poder.

Nos centros de convivência, estudos apontam para o uso de medidas de qualidade de vida a partir da autoavaliação do idoso. "Que nota darei ao dia hoje?" - aumenta a autoestima, o senso de governabilidade e permite a construção de um plano de cuidados singular e a cada dia (HAWKINS, 1988; NERI, 2001; KUCZMARKSI; COTUGNA, 2009; FONSECA, 2010; PASCHOAL, 2011; WICHMANN, 2013).

Tais centros podem proporcionar ao idoso motivo para sair regularmente e oportunidade de contato social (VERAS; CAMARGO JR, 1995). Podem estimular também a formação de uma rede de comunicação em torno do 
cuidado do idoso, construindo uma relação de confiança entre o idoso, seus familiares e os profissionais da instituição. A existência de espaços coletivos para a troca de informaçôes e experiências, bem como os contatos informais do dia a dia contribuem para aproximar os interlocutores. $\mathrm{Na}$ rede de cuidados, o lugar dos diversos atores precisa ser discutido e negociado permanentemente, pois as necessidades e possibilidades do idoso também mudam. Para tanto, os espaços de comunicação, como grupos reflexivos e rodas de conversa, reafirmam a importância da construção coletiva (idosos, cuidadores e profissionais) do cotidiano no espaço. Cabe integrar e formar coordenadores de oficinas a partir de propostas de acompanhamento do envelhecimento humano.

No Berlin Aging Study (BALTES; MAYER, 1999), extenso estudo interdisciplinar realizado entre 1990 e 1993, com 516 idosos de 70-100 anos, selecionados ao acaso da população da cidade de Berlim, foram analisadas diferentes variáveis, abrangendo Medicina (capacidade funcional, perfil de risco, valores biológicos de referência), Sociologia e Políticas Sociais (história de vida e dinâmica geracional, estrutura e dinâmica da família, situação econômica e seguridade social, recursos sociais e participação social) e Psicologia. E no Brasil, alguns portais, como DATASUS, SISAP idoso, censo SUAS e IPEA são referência para informações demográficas sobre esse grupo populacional.

Em centros de convivência, os estudos de coorte podem contribuir para o conhecimento e organização dos cuidados daquele grupo de idosos. Nos Estados Unidos, existem coortes de idosos que envelheceram frequentando centros de convivência (PARDASANI, 2010). As informações epidemiológicas obtidas permitem o monitoramento da saúde e a antecipação de problemas, a fim de ampliar a vida saudável dos idosos (FREITAS; PY, 2011; VERAS et al., 2007; COBERLEY et al., 2011). Nesses centros, múltiplas dimensões do cuidado podem ser observadas - atividades lúdicas e instrumentais.

Programas de promoção da saúde e prevenção de doenças se sucedem no Brasil e no mundo, buscando orientar a atenção à saúde do idoso (ASSIS et al., 2004). Tais abordagens suscitam importantes discussões (CAMARGO JR., 2007; CASTIEL, 2011). Acesso à informaçãa, fatores de proteção e de risco, antecipação, triagens, rastreio, detecção precoce, avaliação geriátrica ampla, avaliações funcional, social (redes) e emocional (vínculos) constituem campo de Saúde Coletiva. 
A avaliação das atividades de vida diária (banho, ida ao vaso sanitário, transferência, continência e alimentação); atividades instrumentais (usar telefone, fazer compras, preparar alimento, controle medicamentos, finanças) e mobilidade (equilíbrio, velocidade de marcha e força em membros) podem contribuir para gerar informações importantes para a tomada de decisões, mapeando fatores de proteção e risco individuais (VERAS et al., 2007).

As atividades médico-sanitárias de educação para saúde podem ampliar seu foco de atenção para dimensões positivas da saúde, para além do controle de doenças específicas. Na revisão Pubmed, os artigos que abordavam prevenção e monitoramento de doenças específicas (n.14/126) discutiam doença oral, obesidade, hipertensão, diabetes, HIV, câncer de próstata ou intestinal, osteoporose, Alzheimer, depressão, prevenção de suicídio, fumo e alcoolismo (NORTHRIDGE et al., 2015).

A triagem auditiva e visual e o auxílio no gerenciamento do uso de múltiplos medicamentos, "a polifarmácia", antecipam a detecção de problemas, contribuindo para o bem-estar do idoso (MOSEGUI, 1999; ROZENFELD, 2003). Hábitos saudáveis, fatores de proteção, incluem alimentação balanceada, prática regular de exercícios físicos, convivência social estimulante, atividade ocupacional e atividades de bem-estar no campo da nutrição (cozinha para diabetes, osteoporose).

$\mathrm{Na}$ revisão Pubmed, muitos artigos abordavam atividades específicas (n. 50/126). Além dos exercícios físicos (n.21/126) (HAMAR et al., 2013), encontramos treino cognitivo (LADITKA et al., 2012), programas nutricionais (FRANCIS et al., 2014), serviços telefônicos, computação, segurança domiciliar, prevenção de quedas, controle de esfíncteres, imunizaçôes e administração financeira. Cuidados com a mobilidade do idoso, prevenção de quedas e equilíbrio em oficinas de psicomotricidade, treinamento de força, orientação na escolha dos sapatos, serviço de podologia são fundamentais, pois garantem a independência.

Envelhecer requer adaptações. Novas aprendizagens são recursos para manter funcionalidade, flexibilidade dos idosos (WICHMANN et al., 2013). Atividades artísticas, culturais e de recreação são tradicionalmente vinculadas a espaços de convivência para idosos e importantes fontes de prazer: conhecimentos gerais, 
idiomas, informática, produção de textos e leitura, arte dos retalhos, dança de salão, teatro, música, jogos de carta, dominó, xadrez, meditação; viagens turísticas. E para o planejamento do próprio envelhecimento, chamamos atenção para um questionário - Time trade-off-que permite uma negociação entre profissional de saúde e idoso, considerando risco e prazer (CAMPOLINA; CICONELLI, 2006).

Muitos idosos aposentados se reinserem no mercado de trabalho de forma regular ou eventual, por prazer ou necessidade, o que representa complementação de orçamento e estímulo ao contato social. Nos Estados Unidos, muitos se comprometem com trabalhos voluntários (PARDASANI, 2010; RILL, 2011).

Centros de convivência podem prestar serviços de assessoria jurídica, agenciamento de cuidadores e auxílio para permanência domiciliar (suporte nas atividades de vida, tele-assistência, entrega de refeiçōes, por exemplo). Para tanto, é fundamental o investimento em cursos para qualificação de cuidadores e na comunicação na rede de cuidados (RABELLO, 2009; CALDAS, 2005).

Ressaltamos que a regularidade de frequência às oficinas permite ao idoso a vivência de uma rotina, o que beneficia também o cuidador, que pode dispor daquele tempo para outras atividades. Um "contrato" semestral ou anual de participação dos idosos às oficinas, segundo desejo deles e disponibilidade de coordenadores, permite melhor gerenciamento. As faltas se devem frequentemente a problemas de saúde ou de transporte. Obstáculo considerável é a dificuldade de deslocamento em metrópoles brasileiras, onde os transportes coletivos não são amistosos para com os idosos (VERAS; CAMARGO JR., 1995; PARDASANI, 2010).

Estes centros também poderiam acolher discussões importantes para o idoso e seu entorno. Envelhecimento e finitude merecem destaque. Podemos nos preparar para envelhecer e morrer? Podemos construir um guia prático, divertido, com antecipações de questôes relevantes do envelhecimento. (O que está acontecendo comigo?).

A filosofia e a religião podem contribuir para a reflexão sobre envelhecimento e morte. Para Platão, na Grécia Antiga, filosofar era aprender a morrer. Idealista, acreditava na vida após a morte, uma passagem, a libertação da alma. Para os materialistas, como Epicuro, a vida era finita e, pela escassez, ela ganhava valor, uma vez que não haveria nada após a morte. Não se vive da mesma maneira quando se acredita em concepçôes tão diferentes de morte (BARROS, 2014). 
Sobre o morrer, muito se tem refletido: momento que vale a pena viver (ARANTES, 2012), motivo de medo. A finitude, os cuidados paliativos e a maneira como culturalmente lidamos com essa fase da vida foram discutidos em trabalho anterior (MOURA, 2001). Ressaltamos a discussão sobre cuidados paliativos, a qualidade no final da vida (HENNENZEL, 1995; HENNENZEL; LELOUP, 1997; HENNENZEL, 2000). Seria possível preparar um guia prático, mas também sensível, apresentando algumas ideias sobre a morte? (Para onde iremos?).

A Psicologia, a partir da discussão sobre vínculos, contribui nessa tarefa. Desapegar da cor dos cabelos, da visão e da audição perfeitas, desapegar da potência, da memória; deixar partir, aceitar o "exoesqueleto" (próteses, aparelhos para audição, óculos, implantes...), criar soluções, transformar e aprimorar. O acolhimento dos medos no grupo é oportuno; entretanto, é tarefa do idoso desapegar-se, viver perdas, dizer adeus. A solidão humana é fato e mesmo necessária. O centro de convivência pode contribuir para a reflexão sobre o sentido da vida, de cada vida, ao cultivar histórias individuais em relatos enriquecidos por fotos, cenas de filmes, músicas, receitas de comida, compartilhando refeições etc.

A trajetória humana ao longo do ciclo vital é construída culturalmente e experimentada de forma singular. Cada fase determina e prediz as possibilidades no momento posterior: envelhecemos como vivemos. Flexibilidade e resiliência (capacidade de fazer face aos acontecimentos) variam de pessoa para pessoa. A experiência de velhice é heterogênea e multidimensional - um convite, portanto, à singularidade também no plano de cuidados.

Ao lidar com os pequenos eventos ou microestressores da vida cotidiana, contamos com recursos pessoais aos quais se agregam recursos sociais. A importância da solidariedade e da troca de experiências é relevante para o idoso e seu entorno. A qualidade ou a funcionalidade do suporte social é mais importante para a adaptação do idoso do que a quantidade de membros da rede e a frequência dos contatos (NERI; FORTES-BURGOS, 2011). E a relação entre suporte social e autocuidado reforça essa visão (HUBBARD et al., 1984; ICHIDA et al., 2013).

Vínculos, desejos e anseios são motores dessa vida. Atenção aos projetos e fomento de novas habilidades que enriquecem o ciclo da vida. 
[...] A vida das gentes neste mundo, senhor Sabugo, é isso.

Um rosário de piscadas.

Cada pisco é um dia.

Pisca e mama;

Pisca e anda;

Pisca e brinca;

Pisca e estuda;

Pisca e ama; Pisca e cria os filhos;

Pisca e geme os reumatismos;

Por fim pisca pela última vez e morre - E depois que morre? - perguntou o Visconde.

- Depois que morre, vira hipótese [...]"

(Emília, em Monteiro Lobato)

Outro fio condutor do acompanhamento do envelhecimento humano é a ancoragem à vida, aos vínculos e ao engajamento em relações significativas: poder contar com familiares e amigos (FONSECA, 2010).

Em pesquisa recente, discutimos a importância da qualidade dos vínculos no início da vida. Os vínculos afetivos dessa fase constituem o alicerce da vida afetiva, indicador da nossa possibilidade de estabelecer outros vínculos significativos seguintes (MOURA, 2013).

Ao longo do ciclo da vida viveremos operações de separação e ligação - corte, costura e transformação - parir, deixar partir, desde o nascimento partimos! Dispositivos culturais e ritos de passagem, como o nascimento, a entrada na escola, a adolescência, a formatura e o casamento, nos ajudam a seguir adiante (FIGUEIREDO, 2009).

O sentido mais profundo de todas as práticas de recepção é o de propiciar para o individuo uma possibilidade de "fazer sentido" de sua vida e das vicissitudes de sua existência ao longo do tempo, do nascimento à morte. Fazer sentido implica estabelecer ligaçôes, dar forma, sequência e inteligibilidade aos acontecimentos. Uma experiência integrada. (FIGUEIREDO, 2009, p. 123).

Contudo, na maior parte das vezes não estamos preparados para dizer adeus: do desapego maior dessa vida, a morte, à infinidade de perdas que constituem o próprio viver. As dimensões do cuidado com o outro, os modos de o agente 
cuidador se apresentar fazem toda a diferença. $\mathrm{O}$ respeito e reconhecimento do outro como diferente de si (alteridade) são fundamentais (BARROS, 2008).

O comprometimento do cuidador (presença implicada), representado pelos fazeres, acolhimento e cuidado ambiental, amplia as possibilidades do cuidado. Entretanto, existe uma forma decisiva do cuidar que não envolve o fazer. É a presença em reserva. No cuidar, é fundamental evitar os excessos de atuação. A presença em reserva diz respeito a ser e deixar ser. Ao cuidador cabe a sabedoria de ausentar-se, ou melhor, manter-se também em presença de reserva. Para isso é necessário que o agente cuidador modere seus fazeres, mantenha-se em reserva e desapegue-se. Ele "deixa ser" seu "objeto" e o não cuidar converte-se em uma maneira muito sutil e eficaz de cuidado, como a da mãe que deixa seu filho brincar sossegado mantendo-se ao lado, silenciosa, dedicando-se, quem sabe, a seus próprios afazeres e interesses. Nesta posição, o cuidador exerce a renúncia à sua própria onipotência (FIGUEIREDO, 2009, 2013).

$\mathrm{O}$ agente dos cuidados melhora a qualidade do que faz quando compartilha seus fazeres e decisões com outros agentes cuidadores. Mais do que isso, ele ganha muito ao descobrir que o objeto de seus cuidados é capaz de exercer funções cuidadoras diante daquele que, supostamente, monopoliza tais tarefas. Deixarse cuidar pelo objeto do cuidado passa a ser, em si mesmo, uma forma eficaz de cuidar (FIGUEIREDO, 2009).

Espaços de convivência propiciam laços afetivos e oportunidades para a expressão de sentimentos. Os registros de histórias, as biografias, fortalecem o idoso, eventualmente demenciado. Ao levá-los a compartilhar suas narrativas com sua rede de cuidadores, favorecemos a construção de um ambiente de comunicação.

Sinto-me intensamente vivo, e quero e espero que, no tempo que me resta, eu possa aprofundar minhas amizades, dizer adeus aos que amo...

Não há tempo para nada que não seja essencial. Minha geração está de saída. ...cada humano é um individuo único, que deve achar seu próprio caminho, viver sua própria vida e morrer sua própria morte. (SACKS, 2015)

\section{Considerações finais}

\section{O lugar do cuidado}

O grupo de idosos está crescendo e envelhecendo, os baby-boomers estão chegando à terceira idade. Cada vez se vive mais e cresce também toda a tecnologia de cuidado 
dessa fase que pode durar 40 anos. É necessário multiplicar e valorizar os espaços de convivência. As soluções são individuais, singulares, mas também coletivas.

Por muito tempo, a instituição "creche" suscitou certa estranheza, por ser associada ao orfanato, local onde crianças eram abandonadas (MOURA, 1989). Instituições que cuidem do idoso durante algumas horas do dia, como o centro de convivência, podem guardar também parentesco indesejável com os asilos, atualmente denominados "instituições de longa permanência" e, algumas vezes, locais de abandono de velhos.

Hoje há consenso em torno da importância da socialização de crianças pequenas. De modo semelhante, evidencia-se o importante papel dos centros de convivência para romper o isolamento social do idoso. Idosos tendem a reduzir seus contatos sociais e seus vínculos. Sua frequência à instituição oferece também ritmo, rotina, organizando um pouco sua vida cotidiana. As redes de sociabilidade do idoso podem acontecer em múltiplos espaços: nas praças, praias, clubes, nas atividades religiosas ou em equipamentos coletivos, como os centros de convivência (FRANÇA; SOARES, 1997; CACHIONI, 1999).

Outros espaços podem também lucrar com as oportunidades de encontros intra ou intergeracionais. As creches, escolas, universidades abertas ou instituições de longa permanência são todos lugares do cuidado, "centros de cuidado", e podem ser continente de intercâmbio geracional (REINSCH; TOBIAS, 1991). Na revisão Pubmed, encontramos um artigo (PARDASANI, 2010) que, ao se referir a modelos inovadores e emergentes, cita o "centro comunitário" destinado a crianças, jovens, adultos e idosos sob o mesmo teto. Promovem recreação, exercícios físicos, refeições, educação, programas intergeracionais e suporte para o cuidador. Com foco em bases multigeracionais, mantêm atividades para cada coorte de idade, mas também têm vários programas e serviços para todos os grupos de idade. Sua missão institucional é ser polo de serviços e programas para toda a comunidade, independentemente de idade. Assim, previne segregação e dá chances àqueles que desejem realizar atividades com outras gerações (PARDASANI, 2010; RILL, 2011).

A instituição que recebe o idoso, temporariamente ou em longa permanência, pode se relacionar em rede, constituindo espaços de convivências. Acolhendo familiares e idosos externos para atividades junto aos idosos residentes, propicia o convívio e a funcionalidade da rede de apoio (POVOA, 2006). Exemplo 
disso é uma experiência alemã que promove o funcionamento, no mesmo edifício, de centro de convivência com atividades diárias para idosos, serviço de saúde (ambulatórios e centro-dia), assistência para permanecer no domicílio e instituição de longa permanência. Prevenir incapacidade e recuperar autonomia com os programas de reabilitação, tudo isso ou algumas combinações destas formas de cuidados no mesmo edifício ampliam o rol de possibilidades. $\mathrm{O}$ quase velho, o jovem velho ou o muito velho experimentam situações aproximadas, que muitas vezes antecipam diferentes estágios de limitações na sua funcionalidade. Multiplicam-se também os recursos, as soluções, mesmo porque a autonomia, a independência nessa fase mudam.

Os "centros de cuidados" poderiam aglutinar cuidados destinados a crianças, pessoas com alguma deficiência temporária ou permanente e idosos, com ou sem autonomia e independência. A creche, ao receber o idoso para atividades junto às crianças, proporciona a convivência e permite construir laços afetivos entre as gerações. A transmissão de valores, as histórias de vida contadas em fotos, receitas de comidas ou canções, a comensalidade, por exemplo, valorizam o idoso, conferindo-lhe um espaço de importância e trazendo motivação. O cultivo dos vínculos, da capacidade de prestar atenção uns aos outros, é consequência natural dessa prática.

Ancorados no instante presente, podemos garantir nosso protagonismo e avaliar cotidianamente nossa disponibilidade para o convívio social, para compartilhar nossa existência. ${ }^{1}$

E você, como está se sentindo hoje? Vamos?

\section{Referências}

AGÊNCIA NACIONAL DE SAÚDE SUPLEMENTAR. Plano de cuidado para idosos na saúde suplementar. Rio de Janeiro: ANS, 2010. 62 p.

ARANTES, C. A morte é um dia que vale a pena viver. Disponível em: <www.youtube.com/ watch?v=ep354ZXKBEs\&feature=youtu.be $>$. Acesso em: 15 jul. 2014.

ASSIS, M.; HARTZ, Z. M. A.; VALLA, V. V. Programas de promoção da saúde do idoso: uma revisão da literatura no período de 1990 a 2002. Ciência e Saúde Coletiva. Rio de Janeiro, v. 9, n. 3, p. 557-581, 2004.

BALTES, P.; MAYER, K. (ed.). The Berlin aging study: aging from 70-100. Londres: Cambridge Press, 1999. 255 p. 
BARROS, C. A morte. Disponível em: <www.youtube.com/watch?v=d5xw-VS1Iuc>. Acesso em: 15 jul. 2014.

BARROS, M. De amoladores de facas e cartógrafos: a atividade do cuidado. In: PINHEIRO, R.; MATTOS, R. (Org.). Cuidar do cuidado: responsabilidade com a integralidade das ações em saúde. Rio de Janeiro: Cepesc, 2008, p.279-295. 44 p.

BRASIL. Ministério da Saúde. Secretaria de Atenção à Saúde. Departamento de Ações Programáticas e estratégicas. Atenção à saúde da pessoa idosa e envelhecimento. Brasília, DF, 2010 (Série Pactos pela Saúde, 12).

CACHIONI, M. Universidades da Terceira Idade: das origens à experiência brasileira. In: NERI, A.; DEBERT, G. (Org.) Velhice e sociedade. Campinas: Papirus, 1999, p. 141-178.

CALDAS, C. (Org.) Valorização da terceira idade: capacitação de acompanhantes de idosos. Rio de Janeiro: UnATI-UERJ, 2005, 169 p.

CAMARANO, A. Demografia e envelhecimento. In: VERAS, R.; LOURENÇO, R. (Org.) Formação humana em geriatria e gerontologia: uma perspectiva interdisciplinar. Rio de Janeiro: UnATI, 2006 p.41-44.

CAMARGO JR., K. As armadilhas da "concepção positiva” de saúde. Physis: Revista de Saúde Coletiva. Rio de Janeiro, v. 17, n. 7, p. 63-76, 2007.

CAMPOLINA, A.; CICONELLI, R. Qualidade de vida e medidas de utilidade: parâmetros clínicos para as tomadas de decisão em saúde. Rev Panam Salud Publica, Washington DC, v. 19, n. 2, p. 128-136, 2006.

CASTIEL, L. D. Utopia/Atopia: Alma-Ata, saúde pública e o "Cazaquistão”. In: CASTIEL, L. D. et al. Das loucuras da razão ao sexo dos anjos. Rio de Janeiro: Fiocruz, 2011. p. 57-72.

COBERLEY, C. et al. Effectiveness of health and wellness initiatives for seniors. Population Health Management, Tennessee, v. 14, suppl. 1, p. s-45-s-50, 2011.

DAL SANTO, T. S. Senior center literature review. Reflecting \& responding to community needs. Sacramento, USA: Archstone Foundation. 2009.

FIGUEIREDO, L. C. As diversas faces do cuidar: considerações sobre a clínica e a cultura. In: MAIA, M. (Org.). Por uma ética do cuidado. Rio de Janeiro: Garamond, 2009 p.121-140. . Cuidado e saúde: uma visão integrada. In: MARIN, I.; ARAGĀO, R. (Org.) Do que fala o corpo do bebê. São Paulo: Escuta, 2013 p.15-36.

FONSECA, M. et al. Papel da autonomia na autoavaliação da saúde do idoso. Rev. Saúde Pública, Rio de Janeiro, v.44 n.1, p.159-65, 2010.

FOUCAULT, M. História da sexualidade, 3: o cuidado de si. Rio de Janeiro: Graal, 1985, 246 p.

FRANÇA, L.; SOARES, N. A importância das relações intergeracionais na quebra de preconceitos sobre a velhice. In: Veras, R. (Org.) Terceira idade: desafios para o terceiro milênio. Rio de Janeiro: Relume Dumará, UnATI/UERJ, 1997, p.143-169. 
FRANCIS, S. L. et al., A theory-based newsletter nutrition education program reduces nutritional risk and improves dietary intake for congregate meal participants. $J$ Nutr Gerontol Geriatr., v. 33, n. 2, p. 91-107, 2014.

FREITAS, E.; PY, L. (Ed). Tratado de geriatria e gerontologia. Rio de Janeiro: Guanabara Koogan, 2011.

GOMES, M.; PINHEIRO, R. Acolhimento e vínculo: práticas de integralidade na gestão do cuidado em saúde em grandes centros urbanos. Revista Interface, Botucatu (SP), v. 9, n. 17, p.287-302, mar-ago, 2005.

HAMAR, B. et al. Impact of a senior fitness program on measures of physical and emotional health and functioning. Popul Health Manag., v. 16, n. 6, p. 364-72, Dec. 2013.

HAWKINS W. E.; DUCAN D. F.; MCDERMOTT R. J. A health assessment of older Americans: some multidimensional measures. Prev Med., v. 17, n. 3, p. 344-56, 1988.

HAWRANIK, P.; PANGMAN, V. Perceptions of a senior citizen's wellness center. The community's voice. J. Gerontol Nurs. Canada, v. 28, n. 11, p. 38-44, 2002.

HENNEZEL, Marie. La mort intime. Paris: Robert Laffont, 1995. 232p.

. LELOUP, J-Y. L'art de mourir: traditions religieuses et spiritualité humaniste face à la mort. Paris: Robert Laffont, 1997. 215 p.

. Nous ne nous sommes pas dit au revoir. Paris: Robert Laffont, 2000.

HUBBARD, P. et al. The relationship between social support and self-care practice. Nurs Res, v. 33, n. 5, p. 266-70, 1984.

ICHIDA, Y. et al. Does social participation improve self-rated health in older population? A quasi-experimental intervention study. SocSciMed., v. 94, p. 83-90, Oct. 2013.

KUCZMARKSI M.; COTUGNA N. Outcome evaluation of a 3-year senior health and wellness initiative. J Community Health, v. 34, n. 1, p. 33-9, Feb. 2009.

LADITKA, J. N. et al. Promoting cognitive health: a web site review of health systems, public health departments and senior centers. Am J Alzheimers Dis Other Demen., v. 27, n. 8, p. 600-8, Dec 2012.

MANDELBAUM, J. Les seniors, acteurs du cinéma. Le Monde, Paris, 7 abr 2013.

MENDES, K. et al. Revisão Integrativa: método de pesquisa para a incorporação de evidências na saúde e na enfermagem. Texto contexto - enferm., v. 17, n. 4, p. 758-764, 2008.

MOLINA, E. et al. Experiencias españolas en la promoción de la autonomia personal en las personas mayores.Gac. Sanit, v. 25, supl., p. 147-157, 2011.

MOSEGUI, G. et al. Avaliação da qualidade do uso de medicamentos em idosos. Revista Saúde Pública, Rio de Janeiro, v. 33, n. 5, p. 437-44, 1999.

MOURA, M.; GUIMARĀES, M.; LUZ, M. Tocar: atenção ao vínculo no ambiente hospitalar. Revista Interface (Botucatu), v. 17, n. 45, p. 393-404, abr/jun 2013. 
MOURA, M. Aspectos psicossociais da situação creche. 1989. 96p. Dissertação (Mestrado em

Medicina Social) - Instituto de Medicina Social, Universidade Estadual do Rio de Janeiro, Rio de Janeiro, 1989.

São muitos os remédios para os males dessa vida: análise de recursos terapêuticos a partir da doença crônica na infância. 2001, 171 p. Tese (Doutorado em Saúde Coletiva) Instituto de Medicina Social, Universidade Estadual do Rio de Janeiro, Rio de Janeiro, 2001. NERI, A. Velhice e qualidade de vida na mulher. In: (Org.) Desenvolvimento e envelhecimento: perspectivas biológicas, psicológicas e sociológicas. Campinas: Papirus, 2001, p.161-200.

NERI, A.; FORTES-BURGOS, A. A dinâmica do estresse e enfrentamento na velhice. In: FREITAS, E.; PY, L. (Org.). Tratado de geriatria e gerontologia. Rio de Janeiro: Guanabara Koogan, 3a ed., 2011. p.1507-1521.

NORTHRIDGE, M. E. et al. A community-based oral public health approach to promote health equity. Am J Public Health, v. 105, suppl 3, p. S459-65, jul. 2015.

ORGANIZAÇÃO MUNDIAL DA SAÚDE. Envelhecimento ativo: uma política de saúde. Brasília,DF: Organização Pan-Americana da Saúde, 2005.59p.

PADARSANI, M.; THOMPSON, P. Senior centers: innovative and emerging models. $J$ Appl Gerontol, v. 29, p. 1-26, 2010.

PASCHOAL, S. M. P. Qualidade de vida na velhice. In: FREITAS, E. V. et al (Org.) Tratado de geriatria e gerontologia. Rio de Janeiro: Guanabara Koogan, 2011. p. 99-106.

PEIXOTO, C. Entre o estigma e a compaixão e os termos classificatórios: velho, velhote, idoso, terceira idade. In BARROS, M. (Org.). Velhice ou terceira idade? Estudos antropológicos sobre identidade, memória e política. Rio de Janeiro: FGV, 2000a, p.69-84.

Envelhecimento e imagem: as fronteiras entre Paris e Rio de Janeiro. São Paulo: Annablume, 2000. 206 p.

PÓVOA, R. Centro de convivência em foco: uma proposta de promoção do envelhecimento através do lazer, da participação social e do intercâmbio geracional. 2006. 90 p. (Trabalho de Conclusão de Curso) - Graduação em Serviço Social, Universidade Federal do Rio de Janeiro, Rio de Janeiro, 2006.

PUTEAUX FRANCE. Mairie de Puteaux, 2015. Disponível em: < http://www.puteaux.fr/ Vie-sociale/CCAS/Les-4-poles-du-CCAS>. Acesso em: 17 mar 2015.

RABELLO, S. Treinamento profissional em RH para o cuidador de idoso e as novas perspectivas de mercado de trabalho no universo do envelhecimento humano. In: BARROS JUNIOR, J. (Org.) Empreendedorismo, trabalho e qualidade de vida na terceira idade. São Paulo: Edicon, 2009. p. 447-485.

REINSCH, S.; TOBIAS J. Intergerational relations: pre-med students at senior centers. Arch Gerontol Geriatr, v. 13, n. 3, p. 211-24. Nov.-Dec. 1991. 
RILL, L. An examination of senior center efficacy: variation in participation and benefits. Dissertação - Universidade da Flórida. Departamento de Sociologia. 2011.

ROZENFELD, S. Prevalência, fatores associados e mau uso de medicamentos entre os idosos: uma revisão. Cad. Saúde Pública. Rio de Janeiro, v. 19, n. 3, p. 717-724, 2003.

SACKS, O. Minha Vida: o neurologista diante da morte. Folha de São Paulo, São Paulo, 22 fev. 2015. Caderno Ilustríssima, p.3.

SOUZA, L.; SILVA, L.; HARTZ, Z. Conferência de Consenso sobre Imagem-objetivo da Descentralização da atenção à Saúde no Brasil. In: HARTZ, Z.; SILVA, L (Org.). Avaliação em Saúde. Salvador: Fiocruz, 2010.

SOUZA, M. T.; SILVA, M. D.; CARVALHO, R. Revisão integrativa: o que é e como fazer. Einstein [Internet], v. 8, n. esp., p. 102-6, 2010. Disponível em: <http://apps.einstein.br/ revista/arquivos/PDF/1134-Einsteinv8n1_p102-106_port.pdf>. Acesso em: 02 nov. 2012.

VERAS, R.P.; CAMARGO JR., K. Idosos e universidade: parceria para a qualidade de vida. In:VERAS, R. P. et al., Terceira idade: um envelhecimento digno para o cidadão do futuro. Rio de Janeiro: Relume-Dumará UnATI/UERJ, 1995. p. 11-27.

VERAS, R. P. et al. Promovendo a saúde e prevenindo a dependência: identificando indicadores de fragilidade em idosos independentes. Revista Brasileira de Geriatria e Gerontologia. Rio de Janeiro, v. 10 n. 3, p. 355-370. 2007.

VERAS, R. P. Estratégias para o enfrentamento das doenças crônicas: um modelo em que todos ganham. Revista Brasileira de Geriatria e Gerontologia. Rio de Janeiro, v. 14, n. 4, p. 779-785, 2011.

. Experiências e tendências internacionais de modelos de cuidado para com o idoso. Ciência\& Saúde Coletiva, Rio de Janeiro, v. 171, n. 1, p. 231-238, 2012 b.

- A necessária gestão qualificada na área de saúde: decorrência da ampliação do segmento etário dos idosos. Jornal Brasileiro de Economia da Saúde, São Paulo, SP, p. 3341, 2012a.

WALLACE, J. et al. Implementation and effectiveness of a community-based health promotion program for older adults. J Gerontol A Biol Sci Med Sci., v. 53, n. 4, p. M301-6, Jul 1998.

WICHMANN, F. et al. Grupos de convivência como suporte ao idoso na melhoria da saúde. Revista Brasileira de Geriatria e Gerontologia, Rio de Janeiro, v. 16, n. 4, p. 821-832, 2013.

WICK, J. Y. Senior centers: traditional and evolving roles. The Consultant Pharmacist USA, v. 27, n. 9, p. 664-7, set. 2012.

\section{Nota}

${ }^{1}$ M.M.D. de Moura foi responsável pela revisão bibliográfica, concepção e redação do artigo, a partir de monografia de conclusão do curso de Especialização em Geriatria e Gerontologia UnATI/UERJ2014. R. P. Veras orientou o estudo que gerou o artigo e realizou a revisão crítica deste texto. 


\section{Abstract}

\section{Monitoring human aging in a care center}

This paper discusses the role of senior centers in human aging. Through literature review we explored history, experiences and possibilities in senior centers. We perceive "seniculture" as cultivation, but also social control of the elderly. Avoiding control, the network care can guide their actions by selfassessment of the elderly and their perception regarding the quality of life. We emphasize the importance of bonds, the caregiver-cared relationship as a space of transformation in the way of dealing with aging. We also propose care center strategies to provides opportunities for intergenerational relations and avoid age-segregation.

Keywords: eldely; health promotion; care center. 